\title{
Two Dimensional Laminar Flow of a Liquid with Circular Hydraulic Jump
}

\author{
Asuquo E.Eyo (Corresponding author) \\ University of Uyo, Nigeria
}

Enobong E. Joshua

University of Uyo, Nigeria

Paul J. Udoh

University of Uyo, Nigeria

Received: February 19, 2011

Accepted: March 22, 2011

doi:10.5539/mas.v5n3p56

\begin{abstract}
We examine two-dimensional laminar flow of a liquid with circular hydraulic jump using boundary layer approach, but with the inclusion of a velocity profile approximated by a quadratic function. Our motivation is due to an earlier work of Watson (1964) on radial spread of a liquid over a horizontal plate. We obtain a new relation for the displacement thickness, momentum thickness and position of the jump. Our approximate values based on Pohlhausen (1921) are compared with the exact values due to Blasius (1908). The comparison shows the error of about $9 \%$ in the shear rate relation on the plate and the error of about $3.5 \%$ in the thickness ratio. Our values agree to a large extent with the exact values and also show improvement of our work upon that of Watson (1964) with respect to the thickness ratio.
\end{abstract}

Keywords: Boundary layer, Circular hydraulic jump, Two-dimensional flow, Velocity profile

\section{Introduction}

It is commonly observed, for example, in a kitchen sink, that a stream of liquid falling vertically on to a horizontal plate spreads out radially in a particular manner. Around the point of impingement there is a circular zone in which a thin layer of liquid moves radially with a high velocity. At the edge of this region the liquid layer abruptly becomes much thicker and consequently flows radially at a much lower velocity. This transformation is called the "hydraulic jump" (Olsson and Turkdogan, 1966 ).

Bush et al. (2006) presented the results of an experimental investigation of the striking flow structures that may arise when a vertical jet of liquid impinges on a thin fluid layer overlying a horizontal boundary. Their experiments revealed a new class of steady asymmetric jump forms that included structures resembling cat's eyes, three - and four - leaf clovers, bowties and butterflies. An extensive parameter study revealed the dependence of the jump on the governing dimensionless groups. The asymmetry-breaking responsible for the asymmetric jumps was interpreted as resulting from a capillary instability of the circular jump.

Kate et al. (2007) used an obliquely inclined circular water jet to impinge on a flat horizontal surface which conferred a series of hydraulic jump profiles, pertaining to different jet inclinations and jet velocities. These jump profiles were non-circular, and could be broadly grouped into two categories based on the angle of jet inclinations made with horizontal. In their work they attemted to find a geometric and hydrodynamic characterization of the spatial patterns formed as a consequence of such non-circular hydraulic jump profiles. Flow-visualization experiments were conducted to depict the shape of demarcating boundaries between supercritical and subcritical flow, and the corresponding radial jump locations were obtained.

Again, Kate et al. (2008) used oblique impingement of a circular liquid jet with a horizontal target to obtain hydraulic jumps of unique non-circular shapes. They investigated experimentally the hydrodynamics of this phenomenon and consequently they observed hydraulic jumps of two broad categories. At higher angles of jet inclination, they noticed that the jumps were bounded by a smooth curve, whereas, at lower angles of inclination, typical jump profiles with corners had been observed. Effect of jet inclination angle on jump profile and jump area had also been investigated. 
Bohr et al. (1993) extended the work on circular hydraulic jump using the shallow water approach. They showed that the circular hydraulic jump could be qualitatively understood using simplified equations of the shallow-water type which included viscosity. They also concluded that it was not possible to determine the position of the jump from ideal theory. Craik et al. (1981) also contributed to the study of circular hydraulic jump using experimental approach. They described new observations of this phenomenon. In addition, they examined a previously unreported instability of the jump and showed this to arise when the local Reynolds number $R_{j}$ just ahead of the jump exceeded a critical value of 147 .

Watson (1964) studied the motion in the thin layer by means of boundary layer theory but with inclusion of a constant velocity profile. He applied the boundary layer theory to the analysis of flow inside the jump and observed that the depth of the flow was much greater on the outside of the jump than on the inside, and hence the condition at the jump might be simplified. The formation of the thin layer and the circular jump was first noticed by Rayheigh (1914) who derived the properties of bores and jumps.

Other contributors include, notably, Belanger(1938), Bouhadef(1978), Felice(2005), Felice and Francesco(2005), Glauert(1958), Groves(2004), Huguera(1994), Kundu and Cohen(2004), Rajput(2006), Rainville and Pinkel(2006), etc.

In this paper our objective is also to discuss circular hydraulic jump by means of boundary layer theory, but with the inclusion of a velocity profile approximated by a quadratic function. Using approximate method certain parameters have been determined and compared with the exact values. Our results agree reasonably with the exact values and also show improvement upon that of Watson with respect to the thickness ratio.

In this analysis, we shall, for convenience, consider the following regions of flow.

(i) The region $0<x<x_{0}$. Here the speed at the edge of the boundary layer is taken as the constant $U_{0}$. When $x<x_{0}$ we find $\delta<h$ and $U(x)=U_{0}$. Here also an approximation to the Blasius type of solution will be derived.

(ii) The region $x>x_{0}$. Here, there is a similarity solution with $\delta=h$ and $U(x)<U_{0}$.

(iii) The region $x=x_{0}$. Here $\delta=h$ and the whole flow is of the boundary layer type.

Note that $x_{0}$ is given by the condition $\delta=h$, so that the whole flow passes through the boundary layer.

\section{Ideal Theory}

The treatment of the problem of two-dimensional flow here applies only to laminar flow in which viscosity is completely neglected. The flow here might be realized by a two-dimensional jet striking a horizontal plane, or by the flow of water under a sluice gate (Glauert,1958). If the flow were realized physically by one of the methods above, $U_{0}$ would be the speed of the impinging jet, or the speed attained by the flow under the sluice gate a short distance downstream of the gate. The ideal or inviscid flow has the uniform depth, a, given by

$$
a=\frac{Q}{U_{0}}
$$

The characteristic Reynolds number is

$$
R=\frac{U_{0} a}{v}=\frac{Q}{v}
$$

Here $Q$ is the volume flux and $v$ the kinematic viscosity. The condition to be applied at the jump (Belanger, 1938) is that the thrust of the pressure is equal to the rate at which momentum is destroyed. If $d$ is the depth outside the jump and $h$ is the depth inside it then the thrust of the pressure per unit length of wave (jump) is

$$
\frac{1}{2} \rho g\left(d^{2}-h^{2}\right)
$$

where $\rho$ is the density and $\mathrm{g}$ is the gravitational acceleration. The speed of flow inside the jump is $U_{0}$ and outside it is $U_{l}$ where

$$
U_{1}=\frac{Q}{d}
$$

The rate of destruction of momentum per unit length of wave is therefore

$$
\rho\left(U_{0}^{2} h-U_{1}^{2} d\right)
$$


Thus

$$
\frac{1}{2} \rho g\left(d^{2}-h^{2}\right)=\rho\left(U_{0}^{2} h-U_{1}^{2} d\right)
$$

Using (1) and (3) in (4) leads to

$$
\frac{1}{2} g\left(d^{2}-h^{2}\right)=Q^{2}\left[\frac{h}{a^{2}}-\frac{1}{d}\right]
$$

When $h<<d$, (5) reduces to (by neglecting $h^{2}$ and $1 / d$ )

$$
\frac{1}{2} g d^{2}=\frac{Q^{2} h}{a^{2}}
$$

which becomes, when $h=a$

$$
\frac{g d^{2}}{2}=\frac{Q^{2}}{a}
$$

A better approximation is to neglect only $\frac{h}{d}$ in (5), so that the pressure thrust inside the wave is ignored but the momentum outside it is included. Thus, from (5) we get

$$
\frac{1}{2} g\left(1-\frac{h^{2}}{d^{2}}\right)=\frac{Q^{2}}{a^{2}}\left(\frac{h}{d^{2}}-\frac{1}{d}\right)
$$

Neglecting $\frac{h^{2}}{d^{2}}$ and setting $\mathrm{h}=\mathrm{a}$ in (8) and then using (7) yields after multiplying the resulting equation by

$$
\begin{aligned}
& \frac{a d^{2}}{Q^{2}} \\
& \frac{g a d^{2}}{2 Q^{2}}\left(1+\frac{a}{d}\right)=1
\end{aligned}
$$

Since $h<<d$, (5) becomes (by neglecting $h^{2}$ )

$$
\frac{g}{2}=\frac{Q^{2} h}{a^{2} d^{2}}-\frac{Q^{2}}{d^{3}}
$$

Putting $h=a$ in (10) and multiplying the result by $\frac{a d^{2}}{Q^{2}}$ leads to

$$
\frac{\operatorname{gad}^{2}}{2 Q^{2}}+\frac{a}{d}=1
$$

Thus, when the depth $h$ is regarded as constant and equal to $a$, the ideal or inviscid theory, identical with the theory of Rayleigh (1914), leaves the position of the jump indeterminate as in Bohr et al.(1993) but gives the results (9), or if the pressure thrust ahead of the wave is neglected it leads to (11).

\section{Blasius Solution of Two Dimensional Laminar Boundary Layer Equations}

Let $x, y$ be the rectangular coordinates with $y$ vertically upwards and $u$, $v$ the corresponding velocity components, then the equations for laminar flow are

$$
\begin{aligned}
& \frac{\partial u}{\partial x}+\frac{\partial v}{\partial y}=0 \\
& u \frac{\partial u}{\partial x}+v \frac{\partial u}{\partial y}=\frac{v \partial^{2} u}{\partial y} \\
& u=v=0 \text { at } \quad y=0
\end{aligned}
$$




$$
\frac{\partial u}{\partial y}=0 \quad \text { at } \quad y=h(x)
$$

$$
\int_{0}^{h(x)} u d y=Q
$$

where $Q$ is the volume flux in the positive x-direction. The total flow from the two-dimensional jet would be $2 Q$, and the flow under the sluice gate is $Q$.

A solution of these equations can be found based on Blasius type of velocity profile given by

$$
u=U_{0} f_{1}^{\prime}(\eta), \quad \eta=\left(\frac{U_{0}}{v x}\right)^{\frac{1}{2}} y
$$

and

$$
f_{1} f_{1}^{\prime \prime}+2 f_{1}^{\prime \prime \prime}=0, \quad(0<\eta<\infty)
$$

is the Blasius equation with boundary conditions

$$
\left.\begin{array}{l}
f_{1}=f_{1}^{\prime}=0 \quad \text { at } \quad \eta=0 \\
f_{1}^{\prime}=1 \quad \text { at } \quad \eta=\infty
\end{array}\right\}
$$

[Here ' denotes differentiation with respect to $\eta]$

Thus the velocity distribution has the Blasius flat-plate profile, and the boundary layer thickness is $\left[\frac{v x}{U_{0}}\right]$. Before considering the approximate solution we shall first find the exact solution of the boundary layer equations (12) - (16).

Thus following Blasius (1908), it follows that

(i) $\left(\frac{v x}{U_{0}}\right)^{\frac{1}{2}} \frac{1}{U_{0}}\left(\frac{\partial u}{\partial y}\right)_{y=0}=f_{1}^{\prime \prime}(0)=0.332$

(ii) The displacement thickness $\delta_{1}$ is

$$
\delta_{1}=1.7208\left(\frac{v x}{U_{0}}\right)^{\frac{1}{2}}
$$

(iii) The momentum thickness $\delta_{2}$ is

$$
\delta_{2}=0.664\left(\frac{v x}{U_{0}}\right)^{\frac{1}{2}}
$$

From (21) and (22) we find

$$
\delta_{1}=2.5915 \delta_{2}
$$

so that the thickness ratio $H^{*}$ becomes

$$
H^{*}=\frac{\delta_{1}}{\delta_{2}}=2.5915
$$

Thus the value of $f_{1}^{\prime \prime}(0)$ in (20) and the value of $H^{*}$ in (24) constitute the exact solution.

\section{Similarity Solution of the Boundary Layer Equations (12) - (13)}

In this section a similarity solution will be derived by direct assumption of the velocity profile approximated by a quadratic function in the form 


$$
u=U(x) f(\eta), \quad \eta=\frac{y}{h(x)}
$$

where

$$
f(\eta)=2 \eta-\eta^{2}
$$

is the similarity profile function.

When the boundary layer finally absorbs the whole flow the velocity profile changes as $x$ increases from the Blasius profile (17) to the similarity profile (25) - (26). Here $U(x)$ is the speed at the free surface $y=h(x)$ and $h(x)$ is the depth of the fluid on the plane. Using the boundary conditions (14) and (15) we find

$$
\begin{aligned}
& f(0)=0, \quad y=0 \\
& f^{\prime}(1)=0, \quad y=h(x)
\end{aligned}
$$

and from (16)

$$
f(1)=1, \quad y=h(x)
$$

Thus $U h$ is a constant, and (12) then leads to

$$
Q=U h \int_{0}^{1}\left(2 \eta-\eta^{2}\right) d \eta
$$

$$
v=U h^{\prime} \eta f(\eta)
$$

or

$$
v=U h^{\prime} \eta\left(2 \eta-\eta^{2}\right)
$$

Using (25) and (29) equation of motion (13) reduces to

$$
v f^{\prime \prime}(\eta)=h^{2} U^{\prime} f^{2}(\eta)
$$

i.e.

$$
v(-2)=h^{2} U^{\prime}\left(2 \eta-\eta^{2}\right)^{2}
$$

from which it follows that $h^{2} U^{\prime}$ is a constant.

Also, $f^{\prime \prime}(\eta) \leq 0$, since the shearing stress $\tau=\mu \frac{\partial u}{\partial y}$ is greatest at the plate, and it is convenient to write

$$
h^{2} U^{\prime}=-\frac{3}{2} k^{2} v
$$

where $k$ is a number.

Using (33) in (32) we find

$$
v(-2)=-\frac{3}{2} k^{2} v\left(2 \eta-\eta^{2}\right)^{2}
$$

or

$$
v .2(-2)=-3 k^{2} v\left(2 \eta-\eta^{2}\right)^{2}
$$

Multiplying (34) by $f^{\prime}=2-2 \eta$, we find

$$
v \cdot 2(-2) \cdot(2-2 \eta)=-3 k^{2} v\left(2 \eta-\eta^{2}\right)^{2} \cdot(2-2 \eta)
$$

or

$$
\frac{\partial}{\partial \eta}(2-2 \eta)^{2}=-k^{2} \frac{\partial}{\partial \eta}\left(2 \eta-\eta^{2}\right)^{3}
$$

Integrating (35) we find.

$$
(2-2 \eta)^{2}=-k^{2}\left(2 \eta-\eta^{2}\right)^{3}+A \quad(A=\text { const })
$$

Using the last two conditions of (27) in (36) we find

$$
A=k^{2}
$$

so that (36) becomes 


$$
\left(2-\eta^{2}\right)^{2}=k^{2}\left[1-\left(2 \eta-\eta^{2}\right)^{3}\right]
$$

Since $\quad 2-2 \eta^{2} \geq 0$, we have from (38)

$$
k=\frac{(2-2 \eta)}{\left[1-\left(2 \eta-2 \eta^{2}\right)^{3}\right]^{\frac{1}{2}}}
$$

Let $t^{\prime}=2 \eta-\eta^{2}$. Then $\frac{d t^{\prime}}{d \eta}=2-2 \eta$. Substituting these in (39), we find

$$
k=\frac{d t^{\prime}}{d \eta}\left(1-t^{\prime 3}\right)^{-\frac{1}{2}}
$$

Separating variables and integrating we obtain

$$
k \eta=\int_{0}^{2 \eta-\eta^{2}}\left(1-t^{\prime 3}\right)^{-\frac{1}{2}} d t^{\prime}
$$

Applying the condition $f(1)=1$ of (27) in (41), we have

$$
k=\int_{0}^{1}\left(1-t^{\prime 3}\right)^{-\frac{1}{2}} d t^{\prime}
$$

Using change of variables $t^{\prime 3}=s,(42)$ becomes

$$
k=\frac{1}{3} \int_{0}^{1} s^{-2 / 3}(1-s)^{-1 / 2} d s
$$

Equation (43) is a well known integral whose solution is written in the form

$$
k=\frac{1}{3} \frac{\Gamma\left(\frac{1}{2}\right) \Gamma\left(\frac{1}{3}\right)}{\Gamma\left(\frac{5}{6}\right)}
$$

where $\Gamma(n)$ is the gamma function.

Now, (39) can be written as

i.e.

$$
k=\frac{\frac{d}{d \eta}\left(2 \eta-\eta^{2}\right)}{\left[1-\left(2 \eta-\eta^{2}\right)^{3}\right]^{\frac{1}{2}}}
$$

$$
k=\left[1-\left(2 \eta-\eta^{2}\right)^{3}\right]^{-\frac{1}{2}} \frac{d}{d \eta}\left(2 \eta-\eta^{2}\right)
$$

Multiplying both sides of (45) by $\left(2 \eta-\eta^{2}\right)$ we find

$$
k\left(2 \eta-\eta^{2}\right)=\left(2 \eta-\eta^{2}\right)\left[1-\left(2 \eta-\eta^{2}\right)^{3}\right]^{-\frac{1}{2}} \frac{d}{d \eta}\left(2 \eta-\eta^{2}\right)
$$

Separating variables and integrating from $\eta=0$ to 1 , (46) becomes

$$
\int_{0}^{1}\left(2 \eta-\eta^{2}\right) d \eta=k^{-1} \int_{0}^{1}\left(2 \eta-\eta^{2}\right)\left[1-\left(2 \eta-\eta^{2}\right)^{3}\right]^{-\frac{1}{2}} d\left(2 \eta-\eta^{2}\right)
$$

Using change of variables $\left(2 \eta-\eta^{2}\right)^{3}=\lambda$ in the rhs of (47), we find

$$
\int_{0}^{1}\left(2 \eta-\eta^{2}\right) d \eta=k^{-1} \int_{0}^{1} \lambda^{\frac{1}{3}}(1-\lambda)^{-\frac{1}{2}} d\left(\lambda^{\frac{1}{3}}\right)
$$


i.e.

$$
\int_{0}^{1}\left(2 \eta-\eta^{2}\right) d \eta=\frac{1}{3} k^{-1} \int_{0}^{1} \lambda^{\frac{1}{3}}(1-\lambda)^{-\frac{1}{2}} d \lambda
$$

Simplifying the rhs of (48) we find

$$
\int_{0}^{1}\left(2 \eta-\eta^{2}\right) d \eta=\frac{1}{3 k} \frac{\Gamma\left(\frac{2}{3}\right) \Gamma\left(\frac{1}{2}\right)}{\Gamma\left(\frac{7}{6}\right)}=\frac{2 \pi}{3 \sqrt{3} k^{2}}
$$

But

$$
\int_{0}^{1}\left(2 \eta-\eta^{2}\right) d \eta=\frac{2}{3}
$$

Therefore, from (49) and (50) we have

$$
\frac{2 \pi}{3 \sqrt{3} k^{2}}=\frac{2}{3}
$$

whence

$$
k=1.34677
$$

Substituting (49) into (28) we find

$$
U h=\frac{3 \sqrt{3} k^{2} \cdot Q}{2 \pi}
$$

We now solve for $U(x)$ and $h(x)$ for the similarity solution using (33) and (52). Now, from (33) we have

$$
U^{\prime}=-\frac{3}{2} k^{2} v \cdot \frac{1}{h^{2}}
$$

Using (52) in (53) we find

$$
U^{\prime}=\frac{d U}{d x}-\frac{3}{2} k^{2} v \frac{4 \pi^{2} U^{2}}{27 k^{4} Q^{2}}
$$

which on separating variables and integrating gives

$$
\frac{1}{U}=\frac{4 \pi^{2} v x}{9 k^{2} Q^{2}}+\text { const } \text {. }
$$

Putting the const $=\frac{4 \pi^{2} v \ell}{9 k^{2} Q^{2}}$ in (55) leads to

$$
\frac{1}{U}=\frac{2 \pi^{2}}{9 k^{2}} \frac{v(x+\ell)}{Q^{2}}
$$

or

$$
U(x)=\frac{9 k^{2}}{2 \pi^{2}} \frac{Q^{2}}{v(x+\ell)}
$$

Here $\ell$ is an arbitrary constant whose presence merely indicates that a shift of origin is possible. Accordingly, substituting (56) into (52) and simplifying we find

$$
h(x)=\frac{\pi}{\sqrt{3}} \frac{v(x+\ell)}{Q}
$$

Equation (56) shows that $U(x)$ varies inversely as $\mathrm{x}$ while (57) shows that $h(x)$ grows in direct proportion to $x$. This is possible within the vicinity of the jump.

\section{Approximate Solution Using Karman- Pohlhausen Method with a Quadratic Function Profile}

Karman-Pohlhausen momentum integral equation for two dimensional laminar flow (see Pohlhausen,1921) is given by 


$$
\frac{\partial}{\partial x} \int_{0}^{\delta}\left(U_{0} u-u^{2}\right) d y=v\left(\frac{\partial u}{\partial y}\right)_{y=0}
$$

while the velocity profile approximated by a quadratic function $(25-26)$ becomes

$$
u=U_{0}\left(2 \eta-\eta^{2}\right), \quad \eta=\frac{y}{\delta(x)}
$$

Here $\delta$ is the boundary-layer thickness.

From (59) we find

$$
\left(\frac{\partial u}{\partial y}\right)_{y=0}=U_{0 .} \frac{2}{\delta(x)}
$$

Now

$\int_{0}^{\delta} U_{0} u d y=U_{0}^{2} \delta(x) \int_{0}^{1}\left(2 \eta-\eta^{2}\right) d \eta=U_{0}^{2} \delta(x) \cdot \frac{2 \pi}{3 \sqrt{3} k^{2}}$

using (49), and

$$
\int_{0}^{\delta} u^{2} d y=U_{0}^{2} \delta(x) \int_{0}^{1}\left(2 \eta-\eta^{2}\right)^{2} d \eta=U_{0}^{2} \delta(x) \cdot \frac{8}{15}
$$

Substituting (60), (61) and (62) into the momentum integral equation (58) we obtain

$$
\frac{\left(10 \pi-8 \sqrt{3} k^{2}\right)}{15 \sqrt{3} k^{2}} U_{0}^{2} \frac{d}{d x} \delta(x)=v U_{0} \cdot \frac{2}{\delta(x)}
$$

Integrating (63) and simplifying we find

$$
[\delta(x)]^{2}=\frac{60 \sqrt{3} k^{2}}{10 \pi-8 \sqrt{3} k^{2}} \cdot \frac{v x}{U_{0}}+C
$$

where $C$ is a constant. If (64) were to remain valid as $x \rightarrow 0$, then $C=0$; or, when $x>>a$ (where a is the uniform depth of the ideal or inviscicid flow) then $C$ could be neglected. Consequently, when $a<<x<x_{0}$,

$$
[\delta(x)]^{2}=\frac{60 \sqrt{3} k^{2}}{\left(10 \pi-8 \sqrt{3} k^{2}\right)} \cdot \frac{v x}{U_{0}}
$$

or

$$
[\delta(x)]^{2}=\frac{60 \sqrt{3} k^{2}}{\left(10 \pi-8 \sqrt{3} k^{2}\right)} \cdot \frac{v x a}{Q},(\text { using }(1))
$$

From (65)

$$
\delta(x)=\left[\frac{v x}{U_{0}}\right]^{\frac{1}{2}}\left[\frac{60 \sqrt{3} k^{2}}{10 \pi-8 \sqrt{3} k^{2}}\right]^{\frac{1}{2}}
$$

Hence

$$
\frac{d \delta}{d x}=\frac{1}{2}\left[\frac{v x}{U_{0}}\right]^{-\frac{1}{2}} \frac{v}{U_{0}}\left[\frac{60 \sqrt{3} k^{2}}{10 \pi-8 \sqrt{3} k^{2}}\right]^{\frac{1}{2}}
$$

Comparing (68) with (63) we have

$$
v U_{0} \cdot \frac{2}{\delta(x)} \cdot \frac{1}{U_{0}^{2}}\left(\frac{15 \sqrt{3} k^{2}}{\left(10 \pi-8 \sqrt{3} k^{2}\right.}\right)=\frac{1}{2}\left[\frac{v x}{U_{0}}\right]^{-\frac{1}{2}} \frac{v}{U_{0}}\left[\frac{60 \sqrt{3} k^{2}}{10 \pi-8 \sqrt{3} k^{2}}\right]^{\frac{1}{2}}
$$

which on simplification yields 
$\left[\frac{v x}{U_{0}}\right]^{\frac{1}{2}} \frac{1}{U_{0}}\left[\frac{\partial u}{\partial y}\right]_{y=0}=2\left[\frac{10 \pi-8 \sqrt{3} k^{2}}{60 \sqrt{3} k^{2}}\right]^{\frac{1}{2}}=f^{\prime \prime}(0)$

(by virtue of (20))

Substituting (51) into (69) we find

$$
\left[\frac{v x}{U_{0}}\right]^{\frac{1}{2}} \frac{1}{U_{0}}\left[\frac{\partial u}{\partial y}\right]_{y=0}=f^{\prime \prime}(0)=0.36514
$$

Substituting the quadratic function profile (25), (26) into the displacement thickness

$\delta_{1}=\int_{0}^{\delta}\left[1-\frac{u}{U_{0}}\right] d y$

we find

$\delta_{1}=\delta \int_{0}^{1}\left[1-\left(2 \eta-\eta^{2}\right)\right] d \eta=\delta\left[1-\frac{2 \pi}{3 \sqrt{3} k^{2}}\right]$

or

$\delta_{1}=\delta\left[\frac{3 \sqrt{3} k^{2}-2 \pi}{3 \sqrt{3} k^{2}}\right]$

Similarly, substituting (25), (26) into the momentum thickness

$$
\delta_{2}=\int_{0}^{\delta} \frac{u}{U_{0}}\left[1-\frac{u}{U_{0}}\right] d y
$$

we have

$$
\delta_{2}=\delta \int_{0}^{1}\left[\left(2 \eta-\eta^{2}\right)-\left(2 \eta-\eta^{2}\right)^{2}\right] d \eta=\delta\left[\frac{2 \pi}{3 \sqrt{3} k^{2}}-\frac{8}{15}\right]
$$

or

$\delta_{2}=\delta\left[\frac{10 \pi-8 \sqrt{3} k^{2}}{15 \sqrt{3} k^{2}}\right]$

Consequently, the thickness ratio $H^{*}$ is

$H^{*}=\frac{\delta_{1}}{\delta_{2}}=\frac{15 \sqrt{3} k^{2}-10 \pi}{10 \pi-8 \sqrt{3} k^{2}}=2.4999$

using (51)

Comparison of the approximate values (70) and (75) with the accurate values (20) and (24) shows that the error in the shear rate relation $f^{\prime \prime}(0)$ is about $9 \%$ while the error in the thickness ratio $H^{*}$ is about $3.5 \%$. The boundary layer just absorbs the whole flow when $x=x_{0}$. When $x<x_{0}$ the total depth $h$ is given by the volume flux condition

$$
U_{0} \delta \int_{0}^{1}\left(2 \eta-\eta^{2}\right) d \eta+U_{0}(h-\delta)=Q
$$

Substituting (50) and (1) into (76) and using the condition $\delta=h$ we have after simplification

$$
h=\frac{3 a}{2}
$$

where $a$ is the jet radius. Since $\delta=h$ when $x=x_{0}$, then using this condition together with (77) and (2) in (67) we obtain 


$$
x_{0}=\frac{9\left(10 \pi-8 \sqrt{3} k^{2}\right) \cdot a R}{240 \sqrt{3} k^{2}}
$$

where $R$ given by (2) is the characteristic Reynolds number. Now the value of $l$ in (56) and (57) can be estimated as follows. Using $x=x_{0}, \delta=h$ and $U(x)=U_{0}$ in (4.31) we find

$U_{0}=\frac{9 k^{2}}{2 \pi^{2}} \frac{Q^{2}}{v\left(x_{0}+\ell\right)}$

which gives, on solving for $\ell$,

$\ell=\frac{9 k^{2}}{U_{0}} \frac{Q^{2}}{2 \pi^{2} v}-x_{0}$

Substituting (78) into (80) and using (1) and (2) we get, after simplification

$$
\ell=\frac{\left[360 \sqrt{3} k^{4}-\left(30 \pi-24 \sqrt{3} k^{2}\right) \pi^{2}\right] a R}{80 \sqrt{3} k^{2} \pi^{2}}
$$

\section{Jump Conditions}

The position $x=x_{l}$ of the hydraulic jump is determined by equating the rate of loss of momentum to the thrust of the pressure. The condition of the momentum is thus

$$
\frac{1}{2} \rho g d^{2}=\rho \int_{0}^{h} u^{2} d y-\rho U_{1}^{2} d
$$

or, using (3)

$$
\frac{1}{2} g d^{2}+\frac{Q^{2}}{d}=\int_{0}^{h} u^{2} d y
$$

Equation (83) is the jump condition. We note that for the case $x_{1}<x_{0}$,

$\int_{0}^{h} u^{2} d y=U_{0}^{2} \delta \int_{0}^{1}\left(2 \eta-\eta^{2}\right)^{2} d \eta+U_{0}^{2}(h-\delta)$ (see ...)

Substituting (84) into (83) the jump condition for the case $x_{1}<x_{0}$ takes the form

$$
\frac{1}{2} g d^{2}+\frac{Q^{2}}{d}=U_{0}^{2} \delta \int_{0}^{1}\left(2 \eta-\eta^{2}\right)^{2} d \eta+U_{0}^{2}(h-\delta)
$$

i.e.

$\frac{1}{2} g d^{2}+\frac{Q^{2}}{d}=U_{0}^{2} \delta \cdot \frac{8}{15}+U_{0}^{2}(h-\delta)$

Using (77) in (86) gives

$$
\frac{1}{2} g d^{2}+\frac{Q^{2}}{d}=U_{0}^{2}\left(\frac{3 a}{2}-\frac{7}{15} \delta\right)
$$

Substituting (67) into (87) we find

$$
\frac{1}{2} g d^{2}+\frac{Q^{2}}{d}=U_{0}^{2}\left[\frac{3 a}{2}-\frac{7}{15}\left[\frac{60 \sqrt{3} k^{2}}{10 \pi-8 \sqrt{3} k^{2}} \cdot \frac{v x}{U_{0}}\right]^{\frac{1}{2}}\right]
$$

Applying (1) to (88) gives

$$
\frac{g d^{2} a^{2}}{2 Q^{2}}+\frac{a^{2}}{d}=\left[\frac{3 a}{2}-\frac{7}{15}\left[\frac{60 \sqrt{3} k^{2}}{10 \pi-8 \sqrt{3} k^{2}} \cdot \frac{v x a}{Q}\right]^{\frac{1}{2}}\right]
$$

Substituting (2) into the rhs of (89) and changing $x$ to $\mathrm{x}_{1}$ we have 


$$
\frac{g d^{2} a^{2}}{2 Q^{2}}+\frac{a^{2}}{d}=\left[\frac{3 a}{2}-\frac{7}{15}\left[\frac{60 \sqrt{3} k^{2}}{10 \pi-8 \sqrt{3} k^{2}} \cdot \frac{x_{1} a}{R}\right]^{\frac{1}{2}}\right]
$$

Solving for $x_{1}$, we obtain

$$
\frac{x_{1}}{a R}=\frac{225}{49}\left[\frac{10 \pi-8 \sqrt{3} k^{2}}{60 \sqrt{3} k^{2}}\right]\left[\frac{3}{2}-\frac{g d^{2} a}{2 Q^{2}}-\frac{a}{d}\right]^{2}
$$

for $x_{1}<x_{0}$.

For the case $x_{1}>x_{0}$ we hav

$$
\int_{0}^{h} u^{2} d y=U_{0}^{2} h \int_{0}^{1}\left(2 \eta-\eta^{2}\right)^{2} d \eta
$$

Substituting (92) into the jump condition (83) we have for the case $x_{1} \geq x_{0}$

$$
\frac{1}{2} g d^{2}+\frac{Q^{2}}{d}=U_{0}^{2} h \int_{0}^{1}\left(2 \eta-\eta^{2}\right)^{2} d \eta
$$

Using (56) and (57) in (93) we find

$$
\frac{v(x+\ell)}{Q^{3}}=\frac{54 \sqrt{3} k^{4}}{15 \pi^{3}}\left[\frac{1}{2} g d^{2}+\frac{Q^{2}}{d}\right]^{-1}
$$

Using (2) in (94) gives

$$
x+\ell=\frac{R Q^{2} \cdot 54 \sqrt{3} k^{4}}{15 \pi^{3}}\left[\frac{1}{2} g d^{2}+\frac{Q^{2}}{d}\right]^{-1}
$$

Finally, substituting (81) in (95) and changing $x$ to $x_{1}$, we obtain

$$
\frac{x_{1}}{a R}=\frac{54 \sqrt{3} k^{4}}{15 \pi^{3}}\left[\frac{g d^{2} a}{2 Q^{2}}+\frac{a}{d}\right]^{-1}-\frac{\left[360 \sqrt{3} k^{4}-\left(30 \pi-24 \sqrt{3} k^{2}\right) \pi^{2}\right]}{80 \sqrt{3} k^{2} \pi^{2}}
$$

\section{Discussion and Conclusion}

From our analysis, the result (11) shows that the position of the jump based on inviscid theory (Bohr te al.,1993) is indeterminate, whereas incorporation of viscous effects (2) in (11) coupled with the use of the principles of momentum and continuity at the jump leads to the modified result (91) for $x_{1}<x_{0}$ or (96) for $x_{1}>x_{0}$. Thus, the position $x=x_{1}$ of the jump is given by (91) for $x_{1}<x_{0}$ or (96) for $x_{1}>x_{0}$. The difference between the inviscid result (11) and the result (91) or (96) due to viscous effects is that (91) or (96) shows that if the left hand side of (11) is less than 1 , the flow loses total head by friction over the length $x_{1}$ until the jump can occur.

In the present work we observe that comparison of the approximate value (70) with the exact value (20) shows that our percentage error in the shear rate relation $f^{\prime \prime}(0)$ on the plate is only about $9 \%$. Similarly, the percentage error in the thickness ratio $H^{*}$ obtained by comparing the approximate value (75) with the accurate value (24) is about 3.5\%. These results are adequate for the present purpose since they closely tend to the accurate values. Also, the result 3.5\% shows improvement of our work upon that of Watson. However the position of the jump (91) or (96) based on viscous effects shows a good correspondence to that of Watson (1964), provided the liquid flow remains laminar. The relation (56) shows that $h(x)$ depends linearly on $x$ while (57) shows that $U(x)$ depends inversely on $x$. Also, (56) means that $x$ starts from the leading edge of the boundary layer, whereas the parameter $\ell$ in this relation is the distance from the centre of the impinging jet to the leading edge of the boundary layer. Finally, the analysis shows that the total thickness of the layer $h(77)$ is directly proportional to $a$, the radius of the impinging jet, implying that $h$ depends chiefly on the model (velocity profile) used.

\section{Acknowledgement}

The authors are greatly indebted to the unknown referees for their helpful suggestions and criticisms.

\section{References}

Belanger, J. B. (1938). Resume de legons. Paris: McGraw-Hill. 
Blasius, H. (1908). Grenzchichten in Flussigluiten mit Kleiner reibung. Zeitschrift für angewandte Mathematik und Physik. 56, English Translation NACA TM No.1256.

Bohr, T., Dimon, P. and Putkaradze, V. (1993). Shallow Water Approach to Circular Hydraulic Jump. Journal of Fluid Mechanics. 254, 635-645.

Bouhadef, M. (1978). Etalement on Couche mince d'un jet liquide cylindrique vertical sur un plan horizontal. Zeitschrift für angewandte Mathematik und Physik. 2 9, 157-168.

Bush, J. W. M., Aristoff, J. M. and Hosoi, A. E. (2006). An Experimental Investigation of the Stability of the Circular Hydraulic Jump. Journal of Fluid Mechanics. 558, 33 - 52.

Craik, A. D. D., Latham, R. C., Fawkes, M. J. and Gribbon, P. W. F. (1981). The Circular Hydraulic Jump.Journal of Fluid Mechanics. 112, 347-362.

Felice, A. (2005). On Nonlinear very Large Sea Wave Groups. Ocean Engineering. 32, 1311-1331.

Felice, A. and Francesco, F. (2005). Nonlinear Space-time Evolution of High Wave Crest. Journal of Offshore Mechanics And Arctic Engineering. 127, No. 1, 46-51.

Glauert, M. B. (1958). Boundary Layer Research (ed. H. Gortler). Belin: Springer Verlag.

Groves, M. D. (2004). Steady Water Waves. Journal of Nonlinear Mathematical Physics. 11, No.4, 435 - 460.

Huguera, F. J. (1994). The Hydraulic Jump in a Viscous Laminar Flow. Journal of Fluid Mechanics. 274, 69-92.

Kate, R. P., Das, P. K. and Chakraborty, S. (2007). Hydraulic Jumps due to Oblique Impingement of Circular Liquid Jets on a Flat Horizontal Surface. Journal of Fluid Mechanics. 573, 247 - 263.

Kate, R. P.,Das, P. K. and Chakraborty, S. (2008). An Investigation on Non-circular Hydraulic Jumps formed due to Obliquely Impinging Circular Liquid Jets. Experimental Thermal and Fluid Science. 32(8), 1429 - 1439

Kundu, P. K. and Cohen, I. M. (2004). Fluid Mechanics (3rd ed.). New York: Elsevier Inc.

Olsson, R.C. and Turkdogan, E. T. (1966). Radial Spread of a Liquid Stream on a Horizontal Plate. Nature. 211, 813-816.

Pohlhausen, K. (1921). Zur naherungsweisen Integration der Differentialgleichung der laminaren Grenzschicht. ZAMM. 1, 252-368.

Rainville, L. and Pinkel, R. (2006). Propagation of Low Mode Internal Waves through the Ocean. Journal of Physical Oceanography. 36, 1220-1236,.

Rajput, R. K. (2006). A Textbook of Fluid Mechanics and Hydraulic Machines. New Delhi: S. Chand.

Rayleigh, L. (1914). On the theory of Long Waves and Bores. Proceedings of Royal Society of London A. 90, p.324.

Watson, E. J. (1964). The Radial Spread of a Liquid Jet over a Horizontal Plate. Journal of Fluid Mechanics. 20, $481-499$.

\section{List of Symbols}

$a=$ Radius of the impinging (incident) jet

$k=$ Parameter in this work $=$ constant

$d=$ Depth of flow outside the wave (jump)

$h=$ Depth of the fluid on the plane $=$ total thickness of the layer

$H^{*}=$ Thickness ratio $=\frac{\delta_{1}}{\delta_{2}}$

$\delta_{1}=$ Displacement thickness

$\delta_{2}=$ Momentum thickness

$f^{\prime \prime}(0)=$ Shear rate relation on the plate

$g=$ Gravitational acceleration

$\ell=$ Arbitrary constant length 


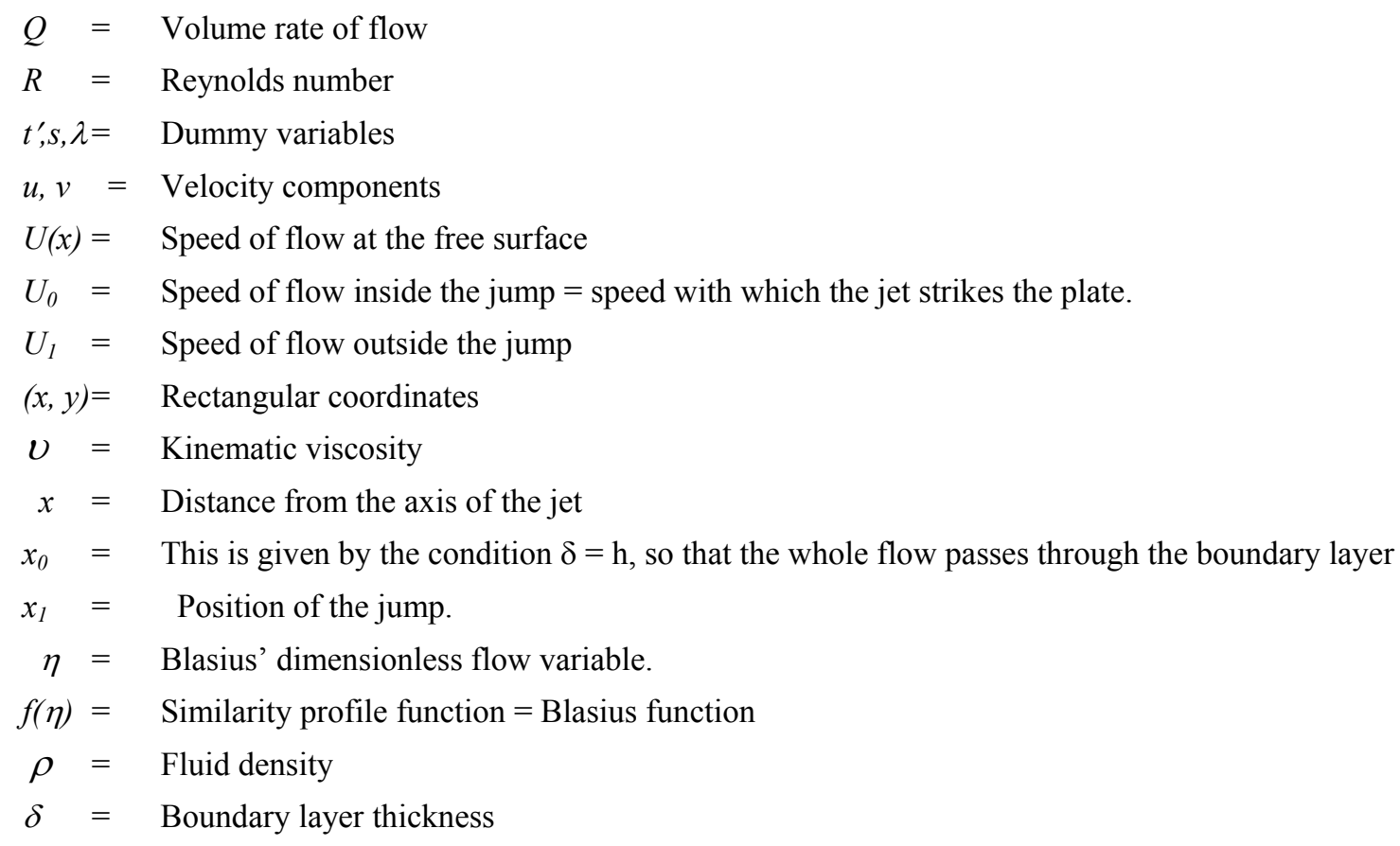

